\title{
Das Kubitaltunnelsyndrom: Diagnostik und Therapieoptionen
}

Frank Unglaub, Peter Hahn, Franziska Kisslinger, Melanie Schäfer, Lars P. Müller, Christian K. Spies

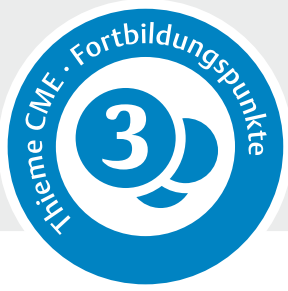

\begin{abstract}
Das Kubitaltunnelsyndrom ist eine Schädigung des N. ulnaris in Höhe des Ellenbogens, verursacht durch eine Kombination aus Kompression, Zug und Reibung. Die Diagnostik basiert auf Anamnese, Inspektion, klinischer sowie neurologischer Untersuchung und ggf. Bildgebung. Die konservativen und operativen Therapieoptionen werden in diesem Beitrag vorgestellt.
\end{abstract}

\section{Einleitung}

\section{Epidemiologie und Ätiologie}

Das Kubitaltunnelsyndrom, im deutschsprachigen Raum auch als Sulcus-ulnaris-Syndrom bezeichnet, ist nach dem Karpaltunnelsyndrom das zweithäufigste Kompressionssyndrom eines peripheren Nervs [3]. Der Begriff Sulcus-ulnaris-Syndrom ist nicht exakt, da die Kompression auch außerhalb des Sulkus zur Druckschädigung des Nervs führen kann. Der Begriff Sulcusulnaris-Syndrom ist im deutschsprachigen Raum aber weit verbreitet.

Der Begriff „Kubitaltunnelsyndrom“ wurde 1958 von Feindel u. Stratford im englischsprachigen Raum eingeführt und fokussiert sich damit auf den Bereich des Ellenbogens als Entstehungsort für dieses Kompressionssyndrom [4].

Das Kubitaltunnelsyndrom ist das zweithäufigste Nervenkompressionssyndrom eines peripheren Nervs. Derzeit geht man von einer Inzidenz von ca. 21 Fällen

\section{DEFINITION}

\section{Kubitaltunnelsyndrom}

N.-ulnaris-Schädigung in Höhe des Ellenbogens, die durch eine Kombination aus Kompression, Zug und Reibung verursacht wird. Unterschieden werden das idiopathische Kubitaltunnelsyndrom, einschließlich Ulnaris(sub)luxation und Kompression durch M. epitrochleoanconaeus ( $\triangleright$ Abb.1), und sekundäre/posttraumatische Formen, auch verursacht durch eine Arthrose des Ellenbogens [1]. pro 100000 Einwohner aus [5]. Männer sind fast doppelt so häufig betroffen wie Frauen, insbesondere bei repetitiver Arbeitsbelastung oder Überkopfarbeit [1]. Beidseitiges Auftreten ist in bis zu einem Drittel der Fälle beschrieben [6].

Das Kubitaltunnelsyndrom ist das häufigste Kompressionssyndrom des N. ulnaris. Die Pathogenese kann sowohl idiopathisch als auch traumatisch, vor allem durch Frakturen des distalen Humerus bzw. im Bereich des medialen Epikondylus, bedingt sein.

Fünf Engstellen im Bereich des medialen Ellenbogens, verursacht durch verschiedene anatomische Strukturen, werden als ursächlich angesehen ( $\triangleright$ Abb. 2).

Im Vordergrund der Kompression steht das sog. „Osborne-Ligament“ (Synonyma: Lig. arcuatum, Lig. epitrochleoanconaeum). Selten wird diese Bandstruktur auch als „Kubitaltunnel-Retinakulum“ bezeichnet. Das Osborne-Ligament zieht vom medialen Epikondylus und dem humeralen Kopf des M. flexor carpi ulnaris zum Olekranon und formt so das Dach des Kubitaltun-
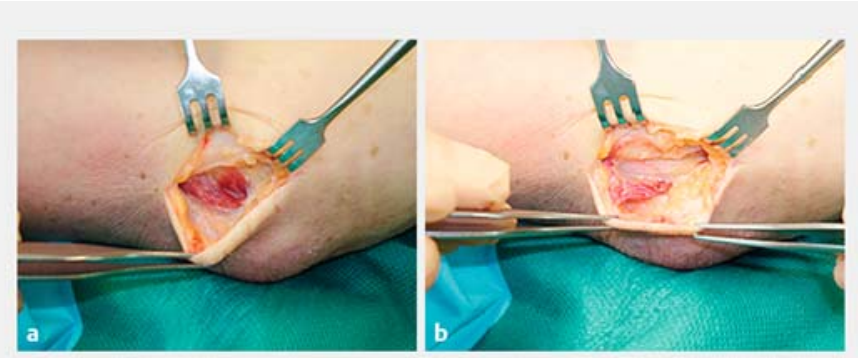

- Abb. 1 M. epitrochleoanconaeus. Der Muskel kann zur Kompression des N. ulnaris führen. Auftreten in ca. $11 \%$ bei Patienten mit Kubitaltunnelsyndrom [2]. 


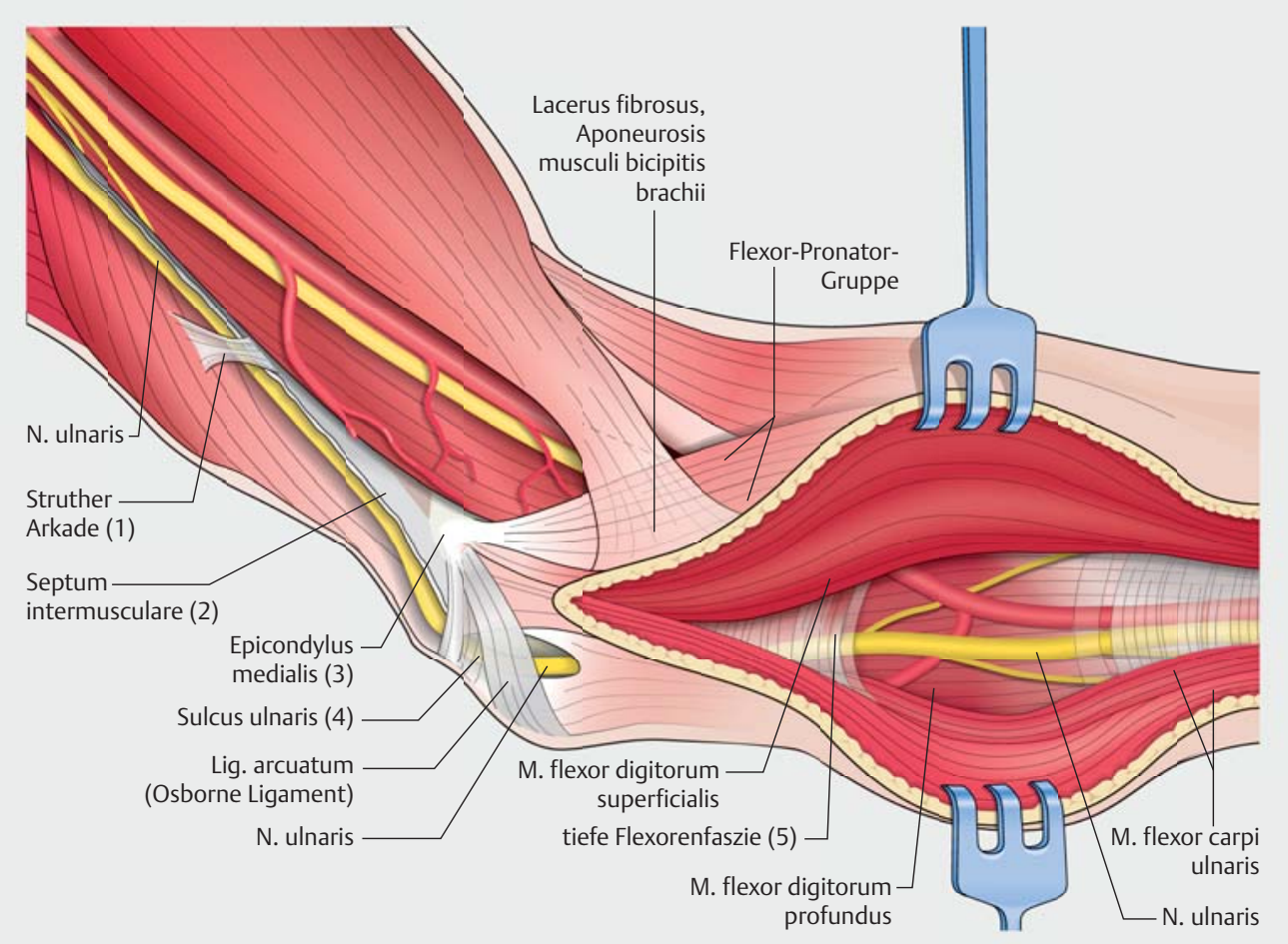

- Abb. 2 Engstellen des Kubitaltunnelsyndroms. 1. Struther-Arkade, ca. $8 \mathrm{~cm}$ proximal des Epikondylus, Verdickung aus Fasern des Septum intermusculare [8], wird kontrovers diskutiert [1]. 2. Septum intermusculare. 3. medialer Epikondylus. 4. Sulcus ulnaris (Osborne-Ligament). 5. Aponeurose des M. flexor carpi ulnaris und pronator teres [7,9].

nels. Das mediale Kollateralband, die Ellenbogengelenkkapsel und weitere Bereiche des Olekranons bilden den Boden des Tunnels [7, 10].

Außerdem kann es zu funktionellen Einengungen kommen. Die Ursache dafür kann eine Verengung des Kubitaltunnels bei Ellenbogenflexion sein. Die Fläche im Tunnel ist in Extension am größten und kann bei voller Ellenbogenflexion um bis zu 55\% abnehmen [11] ( $\triangleright$ Abb.3). Intraneurale Druckuntersuchungen konnten zeigen, dass bei einer Ellenbogenflexion von $40-$ $50^{\circ}$ der Druck am niedrigsten ist und ab $90^{\circ}$ stark ansteigt [12].

An jeder dieser anatomischen Engstellen kann der Nerv komprimiert werden [13].

Letztlich muss meist jedoch von einer multifaktoriellen Entstehung der Ulnariskompression ausgegangen werden. So tragen neben den anatomischen Strukturen auch mechanische Belastungen durch berufliche oder private Tätigkeiten bei, wobei der Effekt von Dauer, Kraft und Stellung der Extremität abhängig ist.

\section{Klassifikation}

Am gebräuchlichsten ist die Einteilung von McGowan mit jeweils 3 Schweregraden (leicht, mittel, schwer) [14] ( Tab. 1).

\begin{tabular}{|l|l|}
\hline $\begin{array}{l}\text { Tab.1 } \\
\text { McGowanteilung des Kubitaltunnelsyndroms nach }\end{array}$ \\
\hline Grad & Beschreibung \\
\hline I & $\begin{array}{l}\text { minimale Läsionen, ohne erkennbare moto- } \\
\text { rische Schwäche der Hand, aber Parästhesien }\end{array}$ \\
\hline II & $\begin{array}{l}\text { intermediäre Läsionen, Muskelschwäche und } \\
\text { Parästhesien vorhanden, aber keine sichtbare } \\
\text { Muskelatrophie }\end{array}$ \\
\hline III & $\begin{array}{l}\text { schwere Läsionen, mit Atrophie von einem } \\
\text { oder mehreren ulnaren intrinsischen Muskeln }\end{array}$ \\
\hline
\end{tabular}




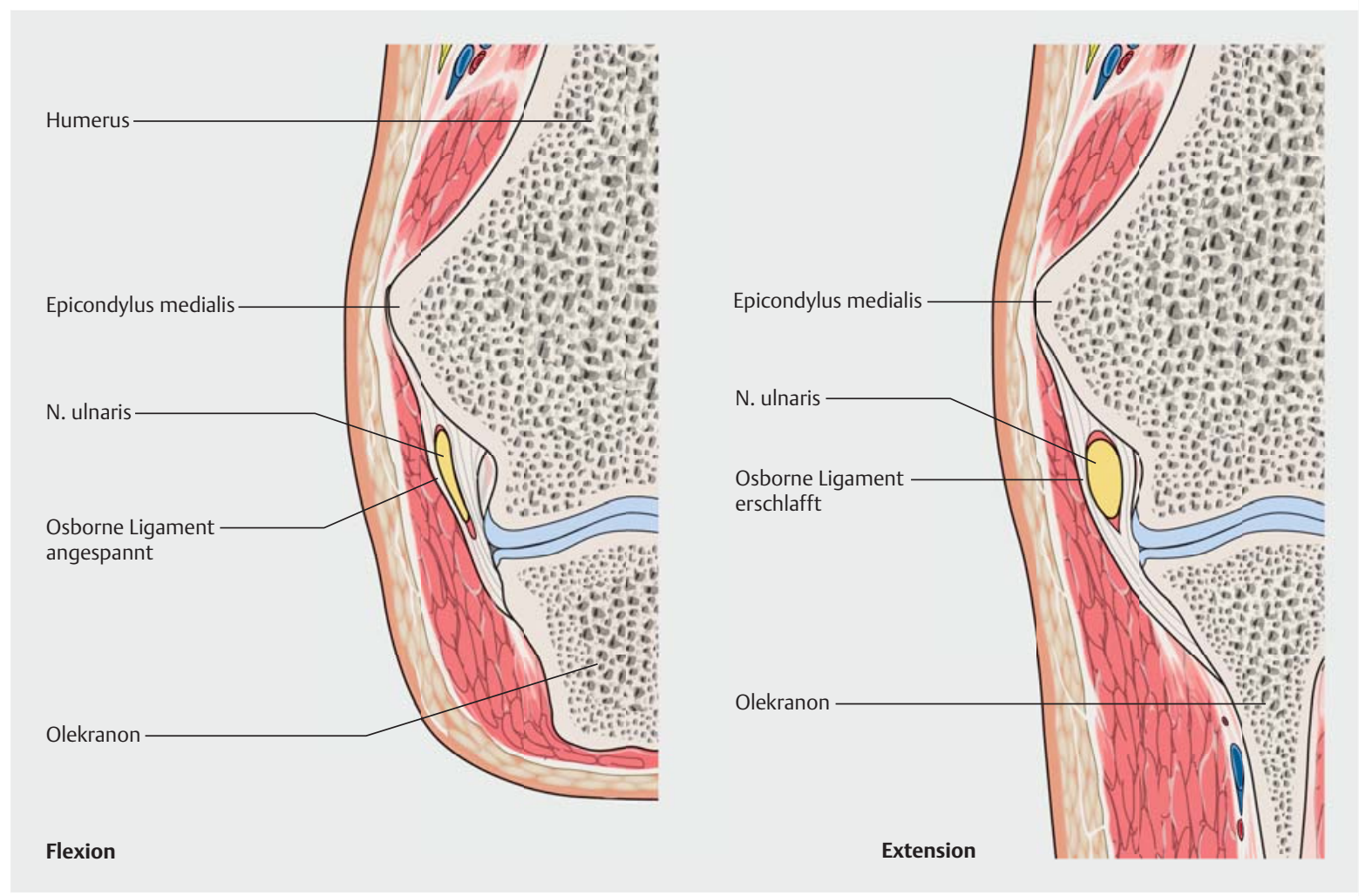

- Abb. 3 Kompression des N. ulnaris durch Flexion im Ellenbogen.

\section{ZUSATZINFO}

\section{Differenzialdiagnosen}

- Loge-de-Guyon-Syndrom: hier jedoch keine Hypästhesie des ulnaren Handrückens, weiterhin Funktion des M. palmaris brevis erhalten

- C8-Radikulopathie: Schmerzen im Schulter-, Skapula- und Halswirbelsäulenbereich, meist weitere Nerven betroffen

- Polyneuropathien (z. B. durch Alkohol, Diabetes mellitus usw.): generalisierte Symptomatik

- amyotrophe Lateralsklerose: Es findet sich nicht nur eine Muskelatrophie der durch den N. ulnaris innervierten Bereiche, sondern meist auch anderer Muskeln; die Atrophie zeigt eine rasche Progredienz

- Thoracic-Outlet-Syndrom: meist weitere Nerven betroffen

- Kamptodaktylie: ähnelt der Krallenhand, wenn Ring- und kleiner Finger betroffen sind. Allerdings ist keine Muskelatrophie oder gestörte Sensibilität nachweisbar [15].

\section{Diagnostik}

Die Diagnose des Kubitaltunnelsyndroms sollte in folgender Reihenfolge durchgeführt werden:

- Anamnese der typischen Symptomatik,

- Inspektion und klinische Untersuchung,

- neurologische Untersuchung der motorischen und sensiblen Nervenleitgeschwindigkeit/Elektromyografie,

- ggf. bildgebende Untersuchung (Röntgen/Ultraschalluntersuchung/Magnetresonanztomografie).

\section{Anamnese, Symptomatik und klinischer Untersuchungsbefund}

Die Beschwerden umfassen die Parästhesie an Ringund Kleinfinger sowie Handrücken und Schmerzen am Ellenbogen, die in Hand oder Schulter ausstrahlen können. Häufig treten die Parästhesien anfallsweise auf, nach entsprechenden Tätigkeiten (z. B. Zeitung lesen, Überkopfarbeit) oder auch nachts.

Außerdem kommt es im Verlauf zu einer deutlichen Kraftminderung, vor allem beim Spitz- und Präzisionsgriff, woraus beispielsweise Schwierigkeiten beim Schreiben resultieren. Auch der Faustschluss (M. flexor digitorum profundus) und die Fingerspreizung ( $\mathrm{Mm}$. interossei) können abgeschwächt sein. 


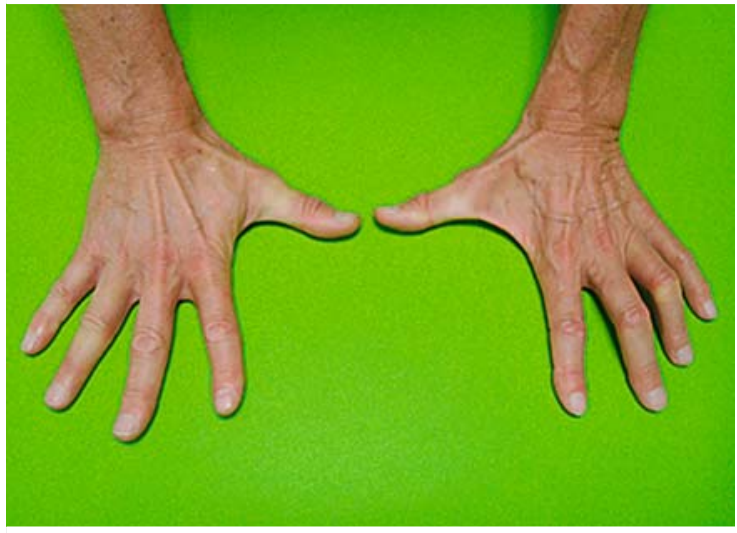

- Abb. 4 Typische Interosseus-Atrophie linksseitig, insbesondere des M. interosseus dorsalis I, und angedeutete Krallenstellung der Finger.

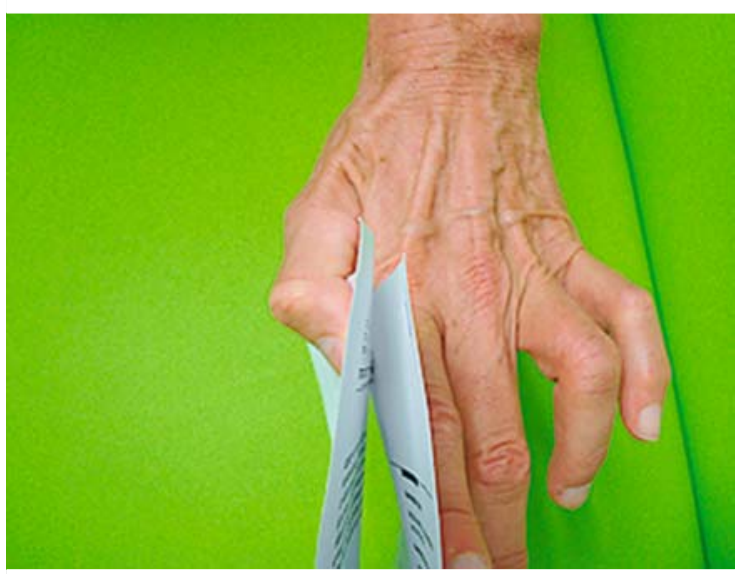

- Abb. 5 Positives Froment-Zeichen. Der Patient versucht die fehlende Adduktion des Daumens zum Festhalten eines Gegenstands durch Flexion des Daumenendglieds zu kompensieren. Der Untersucher versucht hierbei, den Gegenstand vom Patienten weg zu ziehen.

In späten Stadien zeigt der Patient:

- Muskelatrophien, meist besonders auffällig im

1. Interdigitalraum (M. interosseus dorsalis I),

- eine Krallenhandstellung (Ausfall der Interosseusmuskulatur und der Mm. lumbricales),

- ein positives Froment-Zeichen (Ausfall M. adductor pollicis, siehe weiter unten) [1] ( A Abb.4, > Abb.5).

Teilweise steht der Kleinfinger leicht abduziert, dabei handelt es sich um das sogenannte Wartenberg-Zeichen [16].

Bei der Inspektion sollte auf Atrophien, vor allem des M. adductor pollicis geachtet werden. Im fortgeschrittenen Stadium kann auch eine Abspreizung des 5. Fingers oder eine Krallenhand beobachtet werden [17].
Bei der klinischen Untersuchung lässt sich teilweise durch Beklopfen des Nervs proximal des Kubitaltunnels das Hoffmann-Tinel-Zeichen auslösen [18].

Bereits im Frühstadium wird das sogenannte FromentZeichen positiv [17]. Da der durch den N. ulnaris innervierte M. adductor pollicis abgeschwächt ist bzw. ausfällt, werden Gegenstände durch die vermehrte Endgliedbeugung des Daumens (M. flexor pollicis longus) fixiert. Dieser Muskel wird durch den N. medianus innerviert und kompensiert somit die fehlende Adduktion ( $\triangleright$ Abb. 5).

\section{DEFINITION}

\section{Froment-Zeichen}

Als Froment-Zeichen bezeichnet man die kompensatorische Flexion des Daumenendglieds durch den M. flexor pollicis longus, die beim Ausfall des M. adductor pollicis auftritt. Es ist ein Zeichen für die Schwäche/Lähmung des N. ulnaris.

\section{DEFINITION}

\section{Wartenberg-Zeichen}

Das Wartenberg-Zeichen besteht in einer permanenten Abduktion des Kleinfingers aufgrund einer Parese der Hypothenarmuskulatur.

Weiterhin sollte die Sensibilität überprüft werden. Üblicherweise wird die Zwei-Punkte-Diskrimination an Hypothenar und am 4. und 5. Finger im Seitenvergleich gemessen. Dabei ist zu beachten, dass das Innervationsgebiet des N. ulnaris am 4. Finger erheblich variieren kann [19].

Darüber hinaus sollte bei der klinischen Untersuchung nach einer Ulnaris(sub)luxation gefahndet werden. Hierbei palpiert der Untersucher bei Flexion und Extension des Ellenbogens den Nerv im Kubitaltunnel und überprüft seine (Sub)luxationstendenz.

\section{Elektrophysiologische Diagnostik}

Die neurologischen Messungen dienen der Lokalisation des Kompressionssyndroms und dem differenzialdiagnostischen Ausschluss eines Loge-de-Guyon-Syndroms. Außerdem ist so eine Verlaufskontrolle der Therapie möglich [1]. Eine Herabsetzung der motorischen Nervenleitgeschwindigkeit im Ellenbogensegment im Vergleich zum Unterarmsegment um mehr als $16 \mathrm{~m} / \mathrm{s}$ wird als pathologisch gewertet. Hier wird auf neurologische Literatur zum Thema verwiesen [1]. Die elektrophysiologische Untersuchung kann durch eine hochauflösen- 
de Ultraschalluntersuchung des N. ulnaris ergänzt werden.

\section{Bildgebende Diagnostik}

Eine radiologische Untersuchung ist indiziert, um knöcherne Veränderungen vor allem nach Ellenbogentraumata darzustellen [17]. Bei nicht vorhandener Traumaanamnese und unauffälligem klinischen Befund kann auf die bildgebende Diagnostik verzichtet werden.

Primär erfolgt eine Röntgenuntersuchung des Ellenbogens in 2 Ebenen, ggf. zusätzlich eine Tangentialaufnahme zur Einsicht in den Kubitaltunnel. Zur weiteren Abklärung der knöchernen Situation kann die Computertomografie sinnvoll sein. Vermehrt werden auch Magnetresonanztomografie und Sonografie zur Darstellung des Nervs und möglicher Kompressionsursachen eingesetzt [17].

\section{Therapeutisches Vorgehen \\ Konservative Therapie}

Die konservative Therapie kann gute Erfolge bei milden Stadien des Kubitaltunnelsyndroms erzielen. Eine Schulung des Patienten sollte erfolgen, um künftig Tätigkeiten bzw. Haltungen, die mit einer starken Ellenbogenflexion einhergehen, zu vermeiden. Insbesondere direkter Druck auf den Kubitaltunnel sollte unbedingt vermieden werden [12].

Eine Schienenruhigstellung für die Nacht kann verordnet werden, idealerweise in leichter Flexion des Ellenbogens $\left(40-50^{\circ}\right)$. Allerdings ist die Akzeptanz dieser Ruhigstellung oftmals aufgrund des geringen Tragekomforts eingeschränkt. Zusätzlich sollte eine adäquate Schmerzmedikation mit nicht steroidalen Antiphlogistika erfolgen.

\section{Operative Therapie}

Die operative Therapie des Kubitaltunnelsyndroms umfasst verschiedene Verfahren [12], wobei alle Eingriffe unter Lupenbrillenvergrößerung und in Oberarmblutleere durchgeführt werden sollten:

- In-situ-Dekompression,

- endoskopisch-assistierte Dekompression,

- Vorverlagerung des Nervs (submuskulär, intramuskulär, subkutan),

- Epikondylektomie (partiell).

\section{Operationsindikation}

Bei sensomotorischer Ausfallserscheinung und Muskelatrophien sowie fehlgeschlagener konservativer Therapie sollte die Indikation zur operativen Dekompression des N. ulnaris gestellt werden. Auch bei mehrwöchiger pathologischer Verlängerung der Nervenleitgeschwindigkeit in der Elektrophysiologie besteht eine Operationsindikation.

\section{Operationsaufklärung}

- Neben den üblichen Risiken sollte unbedingt die Verletzung von Hautnerven (Läsion des R. cutaneus medialis antebrachii) mit Einschränkung der Sensibilität und Neurombildung mit dem Patienten besprochen werden.

- Auch über einen unvollständigen Operationserfolg, insbesondere bei länger bestehendem Kubitaltunnelsyndrom mit deutlicher Muskelatrophie, sollte der Patient aufgeklärt und eine realistische Prognose gegeben werden.

- Rezidive können bei jeder Operationstechnik auftreten.

- Das Verletzungsrisiko des N. ulnaris ist aufgrund der Narbenbildung bei Revisionseingriffen erhöht.

- Bei längerer Ruhigstellung kann es zu einer eingeschränkten Beweglichkeit des Ellenbogengelenks kommen.

\section{Studienergebnisse}

Mehrere Metaanalysen und systematische Reviews ergaben beim Primäreingriff keine statistisch signifikanten Unterschiede zwischen In-situ- bzw. endoskopischassistierter Dekompression und subkutaner bzw. submuskulärer Vorverlagerung des Nervs [20-23]. Die Vorverlagerung weist jedoch höhere Komplikationsraten auf, wie oberflächliche und tiefe Wundinfektionen, Wunddehiszenz und Asensibilität bzw. Hyposensibilität im Zugangsbereich [24].

Somit wird derzeit die In-situ-Dekompression in offener oder endoskopisch-assistierter Technik als Primäreingriff empfohlen, auch bei (Sub)Luxation des N. ulnaris [1].

Die Vorverlagerung des N. ulnaris wird empfohlen bei:

- posttraumatischer oder degenerativer Deformierung des Ellenbogens,

- ausgeprägter Vernarbung,

- (Sub)Luxation des N. ulnaris mit dadurch verursachter Schmerzsymptomatik [1].

Im Folgenden werden die einzelnen Techniken vorgestellt.

\section{In-situ-Dekompression des Nervus ulnaris}

Die In-situ-Dekompression nach Osborne [25] legt den N. ulnaris oberflächlich durch Längsinzision des Osborne-Ligaments frei. Die Indikation besteht beim Primäreingriff, insbesondere bei kräftigen Patienten mit ausgeprägtem subkutanem Fettgewebe, bei denen eine endoskopisch-assistierte Dekompression erschwert sein kann. Auch als Revisionseingriff ist die In-situ-Dekompression prinzipiell möglich, wobei der Nerv allerdings im vernarbten Gewebe verbleibt. Hierbei erfolgt ein $8-10 \mathrm{~cm}$ langer, bogenförmiger Hautschnitt über dem Epicondylus medialis. 


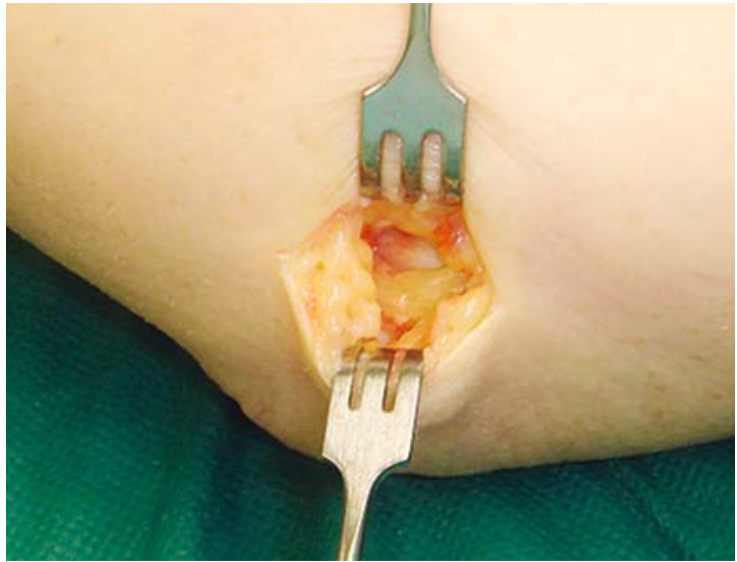

Abb. 6 Darstellung N. ulnaris über eine kurze Hautinzision mit Längsspaltung des Osborne-Ligaments.

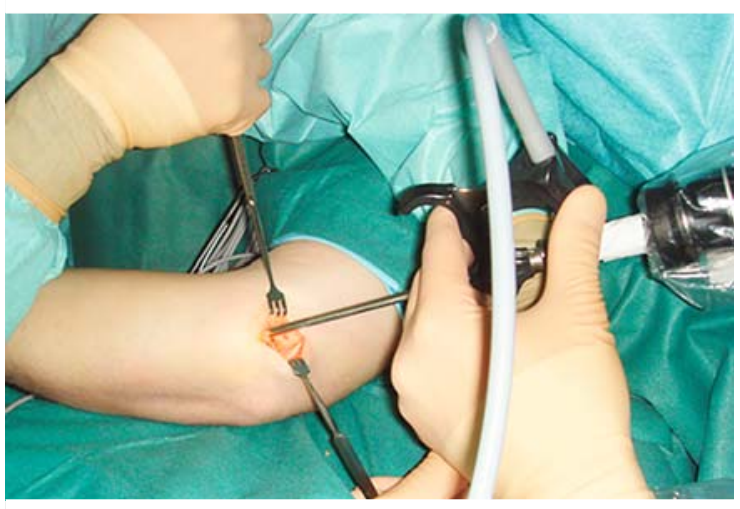

- Abb.7 Endoskopisch-assistierte Dekompression des N. ulnaris mit eingeführtem Dissektor mit konnektierter Optik.

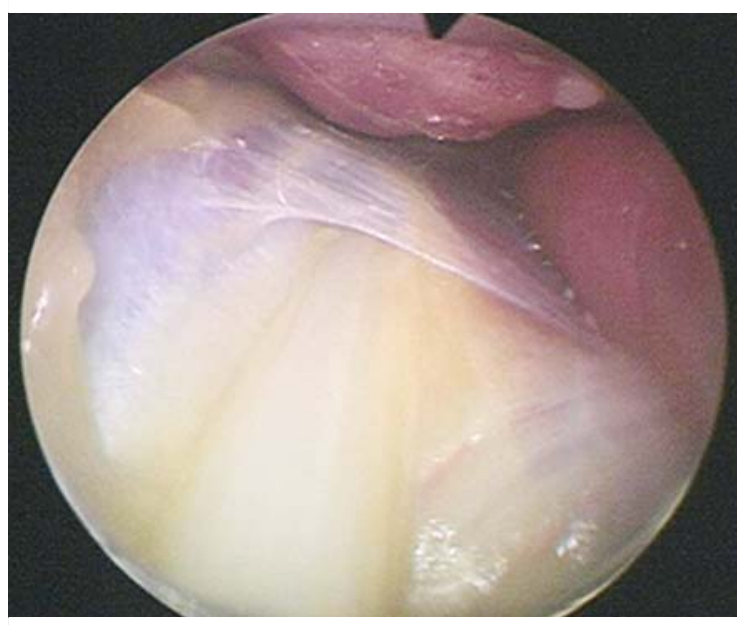

- Abb. 8 Faserzüge der Faszie über dem M. flexor carpi ulnaris $[9,28]$.
Cave

Der Nerv sollte nicht zirkulär aus dem Bett freipräpariert werden, um die Subluxationstendenz nicht zu verstärken und die Vaskularisierung nicht zu kompromittieren, da die Vasa nutriciae entlang des Nervs verlaufen.

Ein Spekulum mit Lichtquelle kann den proximalen und distalen Nervenverlauf optimal ausleuchten und so die Schnittführung verkürzen.

\section{Endoskopisch-assistierte Dekompression des Nervus ulnaris}

Die endoskopisch-assistierte Dekompression des N. ulnaris erfolgt durch eine ca. $1-2 \mathrm{~cm}$ lange Inzision $1 \mathrm{~cm}$ posterior des Epicondylus humeri medialis [26]. Die Indikation besteht beim Primäreingriff. Relative Kontraindikationen sind Revisionseingriffe mit starker Narbenbildung und Patienten mit kräftig ausgeprägtem Subkutangewebe. Unter Schonung des R. cutaneus medialis antebrachii wird das Osborne-Ligament längs eröffnet und der Nerv in der Tiefe dargestellt ( $\triangleright$ Abb.6).

Danach erfolgt die subkutane Tunnelierung nach proximal und distal mit der Kornzange. Nun kann mithilfe eines Spekulums mit Lichtquelle der Nerv weiter nach proximal dargestellt und mit feiner Gewebeschere bzw. Skalpell präpariert werden. Der Dissektor mit konnektierter Optik wird dann nach proximal eingeführt. Es ist dabei darauf zu achten, die Spitze des Dissektors anzuheben, um Platz für das Instrumentieren zu ermöglichen. Mit dem Dissektor kann der Nerv bis zu $10 \mathrm{~cm}$ proximal und distal des Epikondylus dargestellt werden. Mit der Metzenbaumschere erfolgt die Freilegung und Dekompression des Nervs. Für die distale Dekompression wird zunächst die Faszie der beiden Köpfe des M. flexor carpi ulnaris gespalten. Nach der Spaltung kann der Nerv zunächst mit dem Spekulum dargestellt und danach in gleicher Weise mithilfe des Dissektors frei gelegt werden [27] ( $\triangleright$ Abb. 7).

Einschnürende Faserzüge können mit der Metzenbaumschere bis zu $10 \mathrm{~cm}$ distal des Epikondylus durchtrennt werden $(\triangleright \mathbf{A b b} \mathbf{8})$.

Transposition (submuskulär, intramuskulär, subkutan) des Nervus ulnaris

Die Transposition kann submuskulär, intramuskulär oder subkutan erfolgen. Die Indikation ist hauptsächlich beim Revisionseingriff gegeben, um den frei präparierten Nerv aus dem vernarbten Areal in nicht vernarbtes Gewebe zu transponieren. 


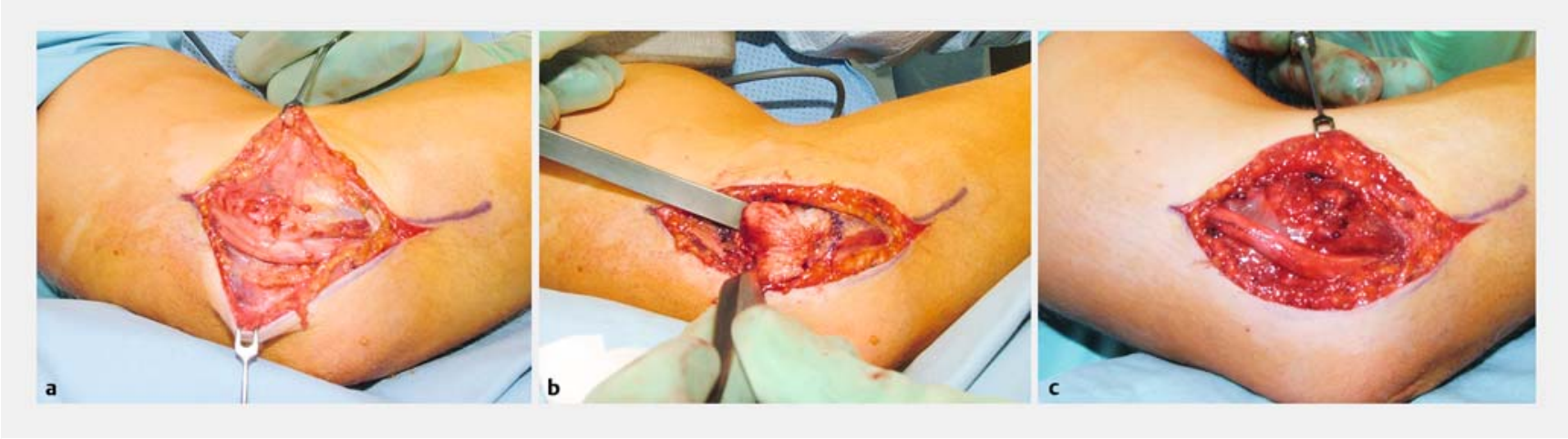

Abb.9 Operative Technik der Epikondylektomie. a Darstellung des N. ulnaris. b Ansetzen des Meisels zur partiellen Epikondylektomie. c Z.n. partieller Resektion und Einnaht eines Periostlappens.

Für die Transposition als Primäreingriff besteht eine Indikation bei:

- posttraumatischer oder degenerativer Deformierung des Ellenbogens,

- ausgeprägter Vernarbung im Nervenverlauf,

- (Sub)Luxation des N. ulnaris mit dadurch verursachter Schmerzsymptomatik.

Die Art der Verlagerung ist abhängig von der Weichteilsituation:

- Bei sehr schlanken Patienten erfolgt in der Regel eine submuskuläre oder intramuskuläre Verlagerung, da subkutanes Fettgewebe nur spärlich vorhanden ist.

- Bei nur geringer Muskelsubstanz erfolgt die submuskuläre Verlagerung anstatt der intramuskulären.

- Bei ausreichend subkutanem Fettgewebe erfolgt die subkutane Verlagerung.

Die Operationstechnik ist bei allen 3 Varianten ähnlich. Nach langstreckiger Darstellung des Nervs erfolgt die komplette Lösung der Anheftungsstellen des Nervs unter Schonung der versorgenden Gefäße. Danach wird die Tasche zur Transposition präpariert. Bei der subkutanen Verlagerung sollte ausreichend Fettgewebe in den Hautlappen eingeschlossen werden, um eine suffiziente Abpolsterung des Nervs zu gewährleisten. Das Septum intermusculare sollte zumindest inzidiert, besser teilweise reseziert werden, um scharfkantige Druckstellen am Nerv zu vermeiden. Bei der Präparation der muskulären Tasche sollte auf eine sorgfältige Blutstillung zur Vermeidung eines postoperativen Hämatoms mit nachfolgender Vernarbung geachtet werden. Hierbei wird die Handgelenkbeugemuskulatur unter Mitnahme der Faszie am Epikondylus scharf abgetrennt. Dies ermöglicht im Nachgang die suffiziente Refixierung der Muskulatur am Ursprung. Der N. ulnaris sollte ausreichend Platz in der Muskeltasche zum Gleiten haben und jede Knickbildung (Kinking) des Nervs sollte vermieden werden. Die subkutane Tasche wird mit resorbierbarem Nahtmaterial locker adaptierend fixiert. Hierbei prüft der Operateur, dass keinerlei Einengungen des Nervs vorliegen.

\section{(Partielle) Epikondylektomie [29]}

Der Zugang erfolgt durch eine ca. $10-15 \mathrm{~cm}$ lange Inzision im Verlauf des N. ulnaris. Die Indikation besteht hauptsächlich beim Revisionseingriff; der Eingriff wird insgesamt aber eher selten durchgeführt. Unter Schonung des $\mathrm{N}$. cutaneus medialis antebrachii wird das Osborne-Ligament dargestellt und längs gespalten. Zunächst wird der N. ulnaris über eine Länge von ca. $10-15 \mathrm{~cm}$ unter Schonung der zirkulären Anheftung und der Gefäßversorgung des Nervs dargestellt. Danach erfolgen die Identifikation des Epikondylus und das Abheben eines halbmondförmigen Periostlappens. Nach partieller Resektion des Epikondylus mit dem Meißel kann durch intraoperative Flexion des Ellenbogens die Gleitfähigkeit des Nervs überprüft werden [30]. Hierbei sollte der Nerv leicht nach anterior gleiten können und nicht im Bereich des Epikondylus „hängen“ bleiben. Nach Refixierung des Periostlappens erfolgt der mehrschichtige Wundverschluss ( $\triangleright$ Abb.9).

\section{Der hypermobile Nerv}

Die Subluxation oder Luxation des N. ulnaris bei Ellenbogenflexion ist in der Normalbevölkerung weit verbreitet, aber nicht mit einem gehäuften Auftreten eines Kubitaltunnelsyndroms assoziiert [31]. Derzeit wird bei hypermobilem N. ulnaris und gleichzeitig bestehendem Kubitaltunnelsyndrom die In-situ-Dekompression empfohlen. Sollte das Luxationsereignis Schmerzen verursachen, dann kann auch die Vorverlagerung des Nervs durchgeführt werden.

\section{Der Revisionseingriff}

Der Revisionseingriff ist häufig durch stark vernarbtes Gewebe, insbesondere im Nervenverlauf, gekennzeichnet. Generell sollte zwischen einem Rezidiv mit verbessertem klinischen Intervall (und verbesserter Elektro- 


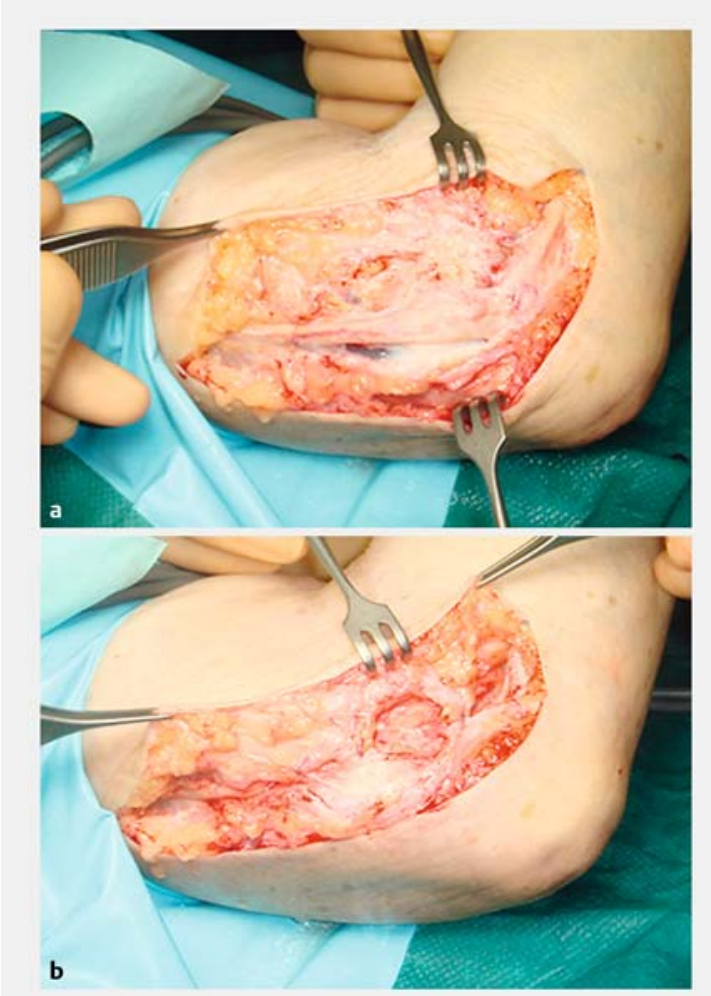

- Abb.10 Revisionseingriff nach vorausgegangener In-situ-Dekompression mit submuskulärer Vorverlagerung des $\mathrm{N}$. ulnaris.

\section{KERNAUSSAGEN}

- Das Kubitaltunnelsyndrom ist eine Schädigung des N. ulnaris in Höhe des Ellenbogens, verursacht durch eine Kombination aus Kompression, Zug und Reibung.

- Die Diagnostik basiert auf Anamnese, Inspektion und klinischer sowie neurologischer Untersuchung. Eine Bildgebung ist insbesondere nach Traumata notwendig.

- Unbehandelt kann das Kubitaltunnelsyndrom zu Sensibilitätsverlust, Muskelschwäche und sekundären Gelenkkontrakturen mit funktioneller Einschränkung führen.

- Während leichte Formen des Kubitaltunnelsyndroms konservativ behandelt werden können, sollte bei Muskelschwäche und ausgeprägter Sensibilitätseinschränkung eine operative Dekompression erfolgen.

- Hierbei unterscheiden sich die klinischen Ergebnisse beim Primäreingriff für die etablierten Techniken wie endoskopisch-assistierte Dekompression, In-situ-Dekompression, subkutane/submuskuläre Verlagerung und die Epikondylektomie nicht voneinander.

- Als Revisionseingriff wird die submuskuläre Vorverlagerung empfohlen, wobei Revisionseingriffe in der Regel aber nicht die guten postoperativen Ergebnisse des Primäreingriffs erreichen. physiologie) und einer inkompletten Dekompression ohne Besserung der klinischen Symptomatik und Elektrophysiologie unterschieden werden. Die Indikation zur Operation besteht bei persistierender (oder nach Intervall wieder) pathologischer Elektrophysiologie und fortbestehender oder wieder aufgetretener neurologischer Einschränkung mit Muskelatrophie ohne Regenerationszeichen. Die Darstellung des Nervs sollte sowohl proximal als auch distal im nicht operierten, narbenfreien Areal beginnen, um eine Nervenläsion zu verhindern. Derzeit wird die Vorverlagerung des Nervs als Revisionsoption empfohlen [32] ( A Abb. 10).

\section{Nachbehandlung}

Die Nachbehandlung richtet sich nach dem operativen Verfahren. Während für minimalinvasive Verfahren wie die endoskopisch-assistierte Dekompression meist eine Bandagierung, die die Flexion im Ellenbogengelenk über $70^{\circ}$ begrenzt, ausreicht, ist bei invasiveren Verfahren wie der Transposition oder Epikondylektomie eine 2-4-wöchige Schienenruhigstellung in leichter Flexion $\left(40-50^{\circ}\right)$ notwendig. Bei verhärteter oder schmerzhafter Narbensituation kann eine Narbenmassage und Narbenabhärtung sinnvoll sein.

\section{Prognose}

\section{Merke}

Wichtigster Prognosefaktor ist die Dauer und Ausprägung des Kubitaltunnels [33]. Je länger und ausgeprägter die Nervenschädigung, desto unwahrscheinlicher ist die komplette Erholung des Nervs und der davon innervierten Muskeln.

Die konservative Therapie erreicht bei milden Stadien des Kubitaltunnelsyndroms und kurzer Symptomdauer gute Ergebnisse. Bei ausbleibender Besserung der Symptomatik nach einer mehrwöchigen konservativen Therapie ist die operative Dekompression indiziert. Bei länger bestehender Parästhesie und beginnenden Kraftverlust kann die operative Therapie, unabhängig vom Operationsverfahren, als Primäreingriff gute Ergebnisse erzielen [1]. Hierbei sollte beachtet werden, dass Atrophien der kleinen Handmuskeln generell ein Zeichen einer schlechteren Prognose sind. Bestehen diese länger als 1 Jahr, bilden diese sich postoperativ meist nicht mehr oder nur unvollständig zurück. Konsekutiv verbleibt eine irreversible Kraftminderung und häufig auch eine Sensibilitätseinschränkung, die sich für den Patienten störend auswirken kann. Durch eine operative Dekompression können aber Muskelatrophien, auch länger bestehend als 2 Jahre, rückläufig sein [1]. Eine komplette Erholung der Muskulatur ist aber unwahrscheinlich. Die Revision eines voroperierten Kubitaltunnelsyndroms erreicht meist nicht die guten Ergebnisse des Primäreingriffs. 
Prof. Unglaub, Prof. Hahn, Dr. Spies sind regelmäßig Referenten bei der Firma Wolf, Knittlingen. Dr. Kisslinger, Frau Schäfer und Prof. Müller geben an, dass kein Interessenkonflikt besteht.

\section{Über die Autoren}

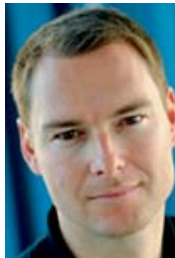

\section{Frank Unglaub}

Prof. Dr. med. Chefarzt Handchirurgie, Vulpius Klinik, Bad Rappenau. Facharzt für Plastische Chirurgie, Zusatzbezeichnung Handchirurgie. Außerplanmäßiger Professor an der Universität Heidelberg. Herausgeber „Archives of Orthopaedic and Trauma Surgery" und „Operative Orthopädie und Traumatologie“.

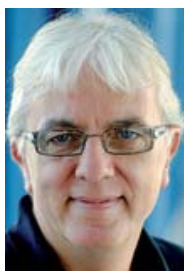

\section{Peter Hahn}

Prof. Dr. med. Chefarzt Handchirurgie, Vulpius Klinik, Bad Rappenau. Facharzt für Plastische Chirurgie, Zusatzbezeichnung Handchirurgie. Außerplanmäßiger Professor an der Universität Heidelberg.

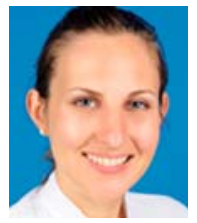

\section{Franziska Kisslinger}

Dr. med. Assistenzärztin Hals-, Nasen-, Ohrenheilkunde, Universitätsklinikum Erlangen (Ärztlicher Direktor: Prof. Dr. med. Iro). Promotion über die klinischen Ergebnisse nach Epikondylektomie bei Prof. Dr. med. Unglaub (Betreuer und Doktorvater) an der Klinik für Plastische- und Handchirurgie am Universitätsklinikum Erlangen (Ärztlicher Direktor: Prof. Dr. med. Horch).

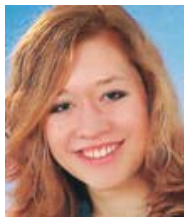

\section{Melanie Schäfer}

Cand. med. Studium der Humanmedizin in Erlangen, Promotion über die klinischen Ergebnisse nach endoskopisch-assistierter Dekompression bei Prof. Dr. med. Unglaub (Doktorvater) und Dr. Spies (Betreuer) an der Medizinischen Fakultät Mannheim der Universität Heidelberg.

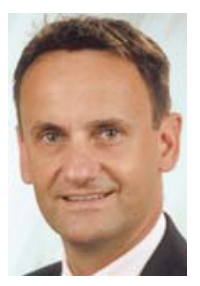

\section{Lars P. Müller}

Prof. Dr. med. Studium der Humanmedizin in Frankfurt und Montreal. 2000-2002 Assistenzarzt Universitätsklinik Mainz, Abteilung für Orthopädie und Unfallchirurgie. 20022007 dort Oberarzt. 2007-2010 dort Leitender Oberarzt. Seit 2011 Leiter der Abteilung Unfallchirurgie an der Universitätsklinik Köln. Fellowships in Boston, Rochester, Hamburg und London.

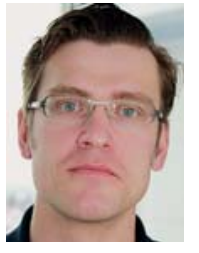

\section{Christian K. Spies}

Dr. med. Oberarzt Handchirurgie, Vulpius Klinik, Bad Rappenau. Facharzt für Orthopädie und Unfallchirurgie, Zusatzbezeichnungen: Handchirurgie, Chirotherapie, Röntgendiagnostik Skelett, Balneologie und physikalische Therapie.

\section{Korrespondenzadresse}

Prof. Dr. med. Frank Unglaub

Handchirurgie

Vulpius Klinik

Vulpiusstraße 29

74906 Bad Rappenau

E-Mail: unglaub@vulpiusklinik.de

Literatur

[1] S3-Leitlinien Diagnostik und Therapie des Kubitaltunnelsyndroms (KUTS).http://www.awmf.org/leitlinien/detail/II/ 005-009.html

[2] Nigst H. Neuropathie des N. ulnaris im Ellenbogenbereich: Operationsbefunde und Folgerungen für den Entstehungsmechanismus und die Operationsindikation. Handchirurgie 1976; 8: $121-124$

[3] Assmus H. Vom Sulcus-ulnaris- zum Kubitaltunnelsyndrom (KuTS). Handchir Mikrochir Plast Chir 2009; 41: 1

[4] Feindel W, Stratford J. Cubital tunnel compression in tardy ulnar palsy. Can Med Assoc J 1958; 78: 351-353

[5] Mondelli M, Giannini F, Ballerini M et al. Incidence of ulnar neuropathy at the elbow in the province of Siena (Italy). J Neurol Sci 2005; 234: 5-10

[6] Artico M, Pastore FS, Nucci F et al. 290 surgical procedures for ulnar nerve entrapment at the elbow: physiopathology, clinical experience and results. Acta Neurochir (Wien) 2000; 142: $303-308$

[7] Palmer BA, Hughes TB. Cubital tunnel syndrome. J Hand Surg Am 2010; 35: 153-163

[8] al-Qattan MM, Murray KA. The arcade of Struthers: an anatomical study. J Hand Surg Br 1991; 16: 311 - 314

[9] Siemionow M, Agaoglu G, Hoffmann R. Anatomic characteristics of a fascia and its bands overlying the ulnar nerve in the proximal forearm: a cadaver study. J Hand Surg Eur Vol 2007; 32: $302-307$

[10] Elhassan B, Steinmann SP. Entrapment neuropathy of the ulnar nerve. J Am Acad Orthop Surg 2007; 15: 672-681

[11] Apfelberg DB, Larson S]. Dynamic anatomy of the ulnar nerve at the elbow. Plast Reconstr Surg 1973; 51: 79-81

[12] Boone S, Gelberman RH, Calfee RP. The Management of Cubital Tunnel Syndrome. J Hand Surg Am 2015; 40: 1897 1904

[13] Bozentka D]. Cubital tunnel syndrome pathophysiology. Clin Orthop Relat Res 1998; 351: 90 - 94

[14] McGowan AJ. The results of transposition of the ulnar nerve for traumatic ulnar neuritis. J Bone Joint Surg Br 1950; 32: $293-301$ 
[15] Frank U, Krimmer H, Hahn P et al. Die chirurgische Therapie der Kamptodaktylie. Handchir Mikrochir Plast Chir 1997; 29: $284-290$

[16] Wartenberg R. Nerves of the Arm: Some of Their Affections; Their Diagnosis. Cal West Med 07 1943; 59: $22-24$

[17] Assmus H, Antoniadis G, Bischoff C et al. Aktueller Stand der Diagnostik und Therapie des Kubitaltunnelsyndroms. Handchir Mikrochir Plast Chir 2009; 41: 2-12

[18] Greenwald D, Blum LC 3rd, Adams D et al. Effective surgical treatment of cubital tunnel syndrome based on provocative clinical testing without electrodiagnostics. Plast Reconstr Surg 2006; 117: 87e-91e

[19] Don Griot JP, Hage J], De Groot PJ. Digital innervation patterns following median or ulnar nerve laceration and their correlation to anatomic variations of the communicating branch between these nerves. J Hand Surg Br 2004; 29: $351-355$

[20] Liu CH, Chen CX, Xu J et al. Anterior Subcutaneous versus Submuscular Transposition of the Ulnar Nerve for Cubital Tunnel Syndrome: A Systematic Review and Meta-Analysis. PLoS One 2015; 10: e0130843

[21] Chen HW, Ou S, Liu GD et al. Clinical efficacy of simple decompression versus anterior transposition of the ulnar nerve for the treatment of cubital tunnel syndrome: A metaanalysis. Clin Neurol Neurosurg 2014; 126: 150 - 155

[22] Macadam SA, Gandhi R, Bezuhly M et al. Simple decompression versus anterior subcutaneous and submuscular transposition of the ulnar nerve for cubital tunnel syndrome: a meta-analysis. J Hand Surg Am 2008; 33: 1314.e1 1314.e12

[23] Zlowodzki M, Chan S, Bhandari M et al. Anterior transposition compared with simple decompression for treatment of cubital tunnel syndrome. A meta-analysis of randomized, controlled trials. J Bone Joint Surg Am 2007; 89: 2591 - 2598

[24] Assmus H, Antoniadis G, Bischoff C. Carpal and cubital tunnel and other, rarer nerve compression syndromes. Dtsch Ärztebl Int 2015; 112: 14-25
[25] Osborne G. Ulnar neuritis. Postgrad Med J 1959; 35: 392 396

[26] Bultmann C, Hoffmann R. Die endoskopische Dekompression des Nervus ulnaris bei Kubitaltunnelsyndrom. Oper Orthop Traumatol 2009; 21: 193-205

[27] Leclère FM, Manz S, Unglaub F et al. [Endoscopic decompression of the ulnar nerve in the cubital tunnel syndrome: about 55 patients]. Neurochirurgie 2011; 57: $73-77$

[28] Folberg CR, Weiss AP, Akelman E. Cubital tunnel syndrome. Part I: Presentation and diagnosis. Orthop Rev 1994; 23: $136-144$

[29] King T, Morgan FP. Late results of removing the medial humeral epicondyle for traumatic ulnar neuritis. J Bone Joint Surg Br 1959; 41: 51 - 55

[30] Schnabl SM, Kisslinger F, Schramm A et al. Objective outcome of partial medial epicondylectomy in cubital tunnel syndrome. Arch Orthop Trauma Surg 2010; 130: 15491556

[31] Calfee RP, Manske PR, Gelberman RH et al. Clinical assessment of the ulnar nerve at the elbow: reliability of instability testing and the association of hypermobility with clinical symptoms. J Bone Joint Surg Am 2010; 92: 2801 - 2808

[32] Nellans K, Tang P. Evaluation and treatment of failed ulnar nerve release at the elbow. Orthop Clin North Am 2012; 43: 487-494

[33] Yamamoto K, Shishido T, Masaoka T et al. Postoperative clinical results in cubital tunnel syndrome. Orthopedics 2006; 29: $347-353$

Bibliografie

DOI http://dx.doi.org/10.1055/s-0042-108428

Handchirurgie Scan 2017; 06: 71-82

(c) Georg Thieme Verlag KG Stuttgart · New York ISSN 2194-8976 


\section{Punkte sammeln auf CME.thieme.de}

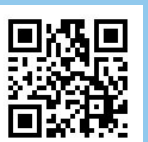

Diese Fortbildungseinheit ist 12 Monate online für die Teilnahme verfügbar.

Sollten Sie Fragen zur Online-Teilnahme haben, finden Sie unter http://cme.thieme.de/hilfe eine ausführliche Anleitung. Wir wünschen viel Erfolg beim Beantworten

der Fragen!

Unter https://eref.thieme.de/ZZWHBY6 oder über den QR-Code kommen Sie direkt zum Artikel zur Eingabe der Antworten.

VNR 2760512017152370740

\section{Frage 1}

Das Kubitaltunnelsyndrom ...

A ist das häufigste periphere Kompressionssyndrom.

B ist immer durch eine Kompression durch die StrutherArkarde verursacht.

C ist meist durch eine Kompression durch das Osborne-Ligament verursacht.

D ist immer durch eine Kompression der Flexor-Aponeurose verursacht.

E wird immer nur durch Flexion verursacht.

\section{Frage 2}

Die Diagnostik des Kubitaltunnelsyndroms beinhaltet ...

A Anamnese, Inspektion, klinische und neurologische Untersuchung.

B nur die Inspektion.

C nur die neurologische Untersuchung.

D nur die Anmanese.

E nur die klinische Untersuchung.

\section{Frage 3}

Die konservative Therapie des Kubitaltunnelsyndroms beinhaltet ...

A eine Nachtlagerungsschiene in maximaler Ellenbogenflexion.

B eine Nachtlagerungsschiene in leichter Flexion $\left(40-50^{\circ}\right)$ des Ellenbogens.

C intensive manualtherapeutische Kompression des Sulcus ulnaris.

D intensive selbstständige Flexion des Ellenbogens durch den Patienten.

E keine antiphlogistische oder Schmerzmedikation.

\section{Frage 4}

Das positive Froment-Zeichen ist gekennzeichnet durch ...

A Abspreizen des Kleinfingers.

B Abspreizen des Zeigefingers.

C Flexion des Daumenendgelenks durch den M. flexor pollicis longus.

D Abspreizen des Mittelfingers.

E Atrophie der Medianus-innervierten Muskulatur.

\section{Frage 5}

Das Wartenberg-Zeichen ist gekennzeichnet durch ...

A Abspreizen des Kleinfingers.

B Abspreizen des Zeigefingers.

C Flexion des Daumenendgelenks durch den M. flexor pollicis longus.

D Abspreizen des Mittelfingers.

E Atrophie der Medianus-innervierten Muskulatur.

\section{Frage 6}

Die Schädigungsstelle des N. ulnaris liegt am häufigsten auf Höhe ...

A der Struther-Arkade.

B des Septum intermusculare.

C des medialen Epikondylus.

D des Sulcus ulnaris (Osborne-Ligament).

E der Aponeurose des M. flexor carpi ulnaris und pronator teres.

\section{Frage 7}

Die Operationsindikation besteht ...

A bei allen Ausprägungen des Kubitaltunnelsyndroms unabhängig der Symptomdauer.

B immer als Notfalleingriff.

C bei sensomotorischer Ausfallserscheinung und Muskelatrophien.

D immer nur bei Muskelatrophie.

E niemals.

\section{Frage 8}

Die Vorverlagerung des N. ulnaris ...

A sollte immer bei Primäreingriff erfolgen.

B sollte niemals bei Revisionseingriff angewandt werden.

C ist gekennzeichnet durch Belassen des Nervs im Sulkus.

D ist gekennzeichnet durch eine kurze Hautinzision von $1-2 \mathrm{~cm}$.

E kann bei Revisionseingriff sinnvoll sein. 


\section{CME-Fragen bei CME.thieme.de}

\section{Fortsetzung ...}

\section{Frage 9}

Der Revisionseingriff bei Kubitaltunnelsyndrom ...

A sollte immer endoskopisch-assistiert erfolgen.

B erreicht immer bessere klinische Ergebnisse als der Primäreingriff.

C ist operativ einfacher durchzuführen als der Primäreingriff.

D kann durch eine Nervenverlagerung erfolgen.

E weist intraoperativ niemals eine langstreckige Vernarbung des Nervs auf.

\section{Frage 10}

Die Nachbehandlung des operativ therapierten Kubitaltunnelsyndroms ...

A beinhaltet immer die 6-wöchige Gipsruhigstellung.

B beinhaltet immer die Ruhigstellung in voller Streckstellung des Ellenbogens.

C ist abhängig von der angewandten Operationstechnik.

D beinhaltet niemals ein Narbenabhärtungstraining.

E beinhaltet immer die 12-wöchige Ruhigstellung. 